$\begin{array}{llll}\text { Submission: 14/02/2019; } & 1^{\text {st }} \text { round notif.: 28/05/2019; } & \text { New version: 13/06/2019; } & 2^{\text {nd }} \text { round notif.: 02/07/2019 } \\ \text { Camera ready: 29/08/2019; } & \text { Edition review: 30/10/2019; } & \text { Available online: 16/02/2020; } & \text { Published: 16/02/2020 }\end{array}$

\title{
Graphical User Interface for educational content programming with social robots activities and how teachers may perceive it
}

\author{
Daniel Carnieto Tozadore \\ Institute of Mathematics and Computer Sciences \\ University of São Paulo \\ tozadore@usp.br
}

\author{
Roseli Aparecida Francelin Romero \\ Institute of Mathematics and Computer Sciences \\ University of São Paulo \\ rafrance@icmc.usp.br
}

\begin{abstract}
Interactive devices have been successfully applied in education in the last decades. The most used devices for such tasks are personal computers and tablets, due to its financial trade-off and popularization. Social robots are less used, mainly because of their cost and the complexity of being programmed. In this paper, a solution to work around the complexity of programming social robots is presented as a Graphical User Interface (GUI). The GUI system controls an interactive robot which plays with the students and adapts its behavior autonomously. During the activity execution, the adaptive algorithm detects student's body signals and verbal responses to adapt the addressed content to harder or easier questions. After creating and running an activity, all sessions' evaluation and information can be accessed for visual analysis, as well as students' preferences throughout the interaction. The proposal was presented to regular teachers from the elementary school that answered a questionnaire about their perception of this proposal. The answers were analyzed and, in general, they seemed to slightly notice the system potential in and how it can support them in after-classes exercises, despite it requires some time to fully get used with the interface.

Keywords: Human-Robot Interaction; Graphic User Interface; Educational Tool, Educational Robotics
\end{abstract}

Cite as: Tozadore, D. C., \& Romero, R. A. F. (2020). Graphical User Interface for educational content programming with social robots activities and how teachers may perceive it. Brazilian Journal of Computers in Education (Revista Brasileira de Informática na Educação - RBIE), 28, 191-207. DOI: 10.5753/RBIE.2020.28.0.191 


\section{Introduction}

Social robots are the robots that are capable of changing information between themselves or with humans (Goodrich \& Schultz, 2007). They are widely used in several tasks from entertainment to medicine (Leite, Martinho, \& Paiva, 2013). However, social robots are far from achieving popularization due to the high costs and lack of people's knowledge about designing and programming robots. Because of that, smartphones and tablets are the most common electronic devices used in learning activities, for being the opposite of the robots in this sense: they have low cost and a consolidate familiarization with the users. Mainly, in the educational field, the lack of training of teachers and their inclusion in the robot's programming are highlighted as one important concern in a worldwide scenario. Some authors pointed out this factor as an even more critical problem than the robot's higher costs (Johal, Castellano, Tanaka, \& Okita, 2018).

Research that considers social robots to achieve success in tasks with humans are placed in the Human-Robot Interaction (HRI) field. These types of applications are known for increasing people's curiosity and motivation in social and intellectual activities because interactive robots are not common in our daily lives. Nonetheless, after the robot loses its novelty and the users get used them, a decrease in the users' motivation and attention span is commonly noticed. A robot programmed with memory about the users, personalized conversations and adaptation of content difficulty can hold students' interest in the pedagogical interactions for a longer period, compared to robots with simple and monotonous behavior. By searching in the literature, several types of research work can be found on building an efficient adaptive system (Belpaeme et al., 2018a). But a lack of studies to improve content programming effortlessness was noted. It means few social robotic systems allow non-programmers to design and execute HRI activities.

This paper is an extension of the published work entitled "Graphical User Interface for Adaptive Human-Robot Interaction Design in Educational Activities Creation", in the XXIX Simpósio Brasileiro de Informática na Educação (SBIE). The first version presented the Graphical User Interface (GUI) components and information that would be more helpful to the teachers, such as dialogue, content, and student and evaluation databases. This extended version presents also some system's implementation decisions and interfaces functionalities that would be more relevant to programmers, such as the vision subsystem and their methods, the adaptation algorithm and the interaction interface. It also deliveries an unprecedented study with regular teachers from the elementary school. They participate in a presentation about the proposed system and they were inquired about their opinion of how much this type of solution can be helpful to them in afterclasses exercises.

The robotic architecture is a cognitive adaptive system (Tozadore et al., 2017) that encapsulates a module-based implementation to run over the robot's sensors and actuators. The interaction flow overview provided by this architecture is shown in Figure 1. The person who designs the interactions (the designer) can program the activities in the system's GUI. The designer is represented as the teacher in this illustration since it is expected that the teachers mainly perform this role. During the activities execution, the system controls the robot to autonomously interact and adapt to the student. 


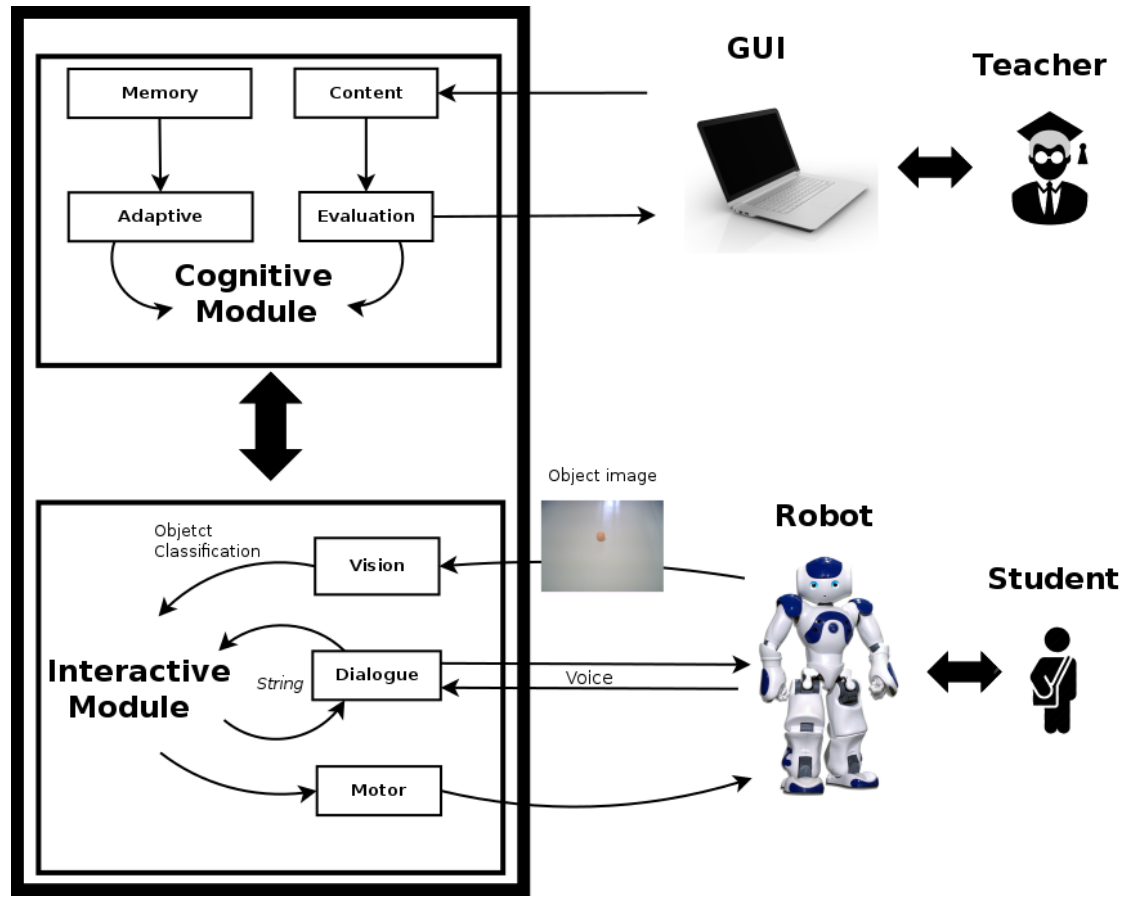

Figure 1: Architecture scheme.

The system interface with the student is a NAO robot, from Softbank robotics ${ }^{1}$ due to being part of the available materials of the researcher institution. However, any interactive device with microphone, screen and speakers can be used with this system, after the right calibration. NAO is a $60 \mathrm{~cm}$ tall humanoid robot that has visual and sound resources (among others) designed to interact with humans in general purposes. Its application in educational tasks has been explored in several studies and shown well accepted.

\section{Related Work}

The predominance of mountable kits as Lego Mindstorms ${ }^{2}$ and Pete ${ }^{3}$ are often highlighted in the literature (Mubin, Stevens, Shahid, Al Mahmud, \& Dong, 2013). They offer intuitive graphical interfaces to the users and they are most of the applications used as educational robotics (Benitti, 2012). However, their usage is limited to the STEM field ${ }^{4}$ domain. After programming, the robots execute the student's code and there is no interaction between them. Hence, they are perceived by the students more than a learning tool than an agent that can play an active role in their cognitive process. Whereas social robots are not programmed by the students, but they are more supportive during the activities and capable to cover topics for more areas (Belpaeme et al., 2018b) due to the capability of verbal communication.

The development of systems that adapt to the students' difficulty is something commonly aimed in technological teaching designs. The Intelligent Tutoring Systems (ITS) are systems which adapt to the student necessities. They are often used in electronic learning and its implementation may vary from one application to another (Murray, 1999). Their usage increases the students' learning experience and provides better consequences in the content fixation. In general, the ITS also provide to the teachers an easy approach to plan the activity to be performed through GUI (Paiva, 2017). Nonetheless, embodied systems provide a more complete experience

\footnotetext{
${ }^{1}$ www.softbankrobotics.com

2 www.lego.com/en-us/mindstorms

${ }^{3}$ www.pete.com.br

${ }^{4}$ Science, Technology, Engineer and Mathematics
} 
than virtual learning environment and few of the existing ITS can be used along social robots (Platz, Krieger, Niehaus, \& Winter, 2018). Those whose made use of ITSs with robots claimed they witness significant enhance in the young student's learning rate. These contributions go from pronunciation skills (Spaulding, Chen, Ali, Kulinski, \& Breazeal, 2018) to Mathematics (Clabaugh et al., 2017). Moreover, GUI's allowed the settings of educational robotics that are not sociable to be more intuitive. Thus, it is expected to be helpful in this scenario as well (Rivas et al., 2015).

Among the advantages of social robots, one that can be highlighted is the advantage to aggregate human relation characteristics to the process such as shape and personalization of the robot. For instance, personalization in social interactions has shown to be an alternative in keeping the engagement of the users (Lucas et al., 2018). In the same way, by simulating the feeling of rapport building between robot and user, is possible to explore social techniques to enhance the results in the performed activity (Lucas et al., 2018).

Shiomi et al. (2006) investigated the influence of the free-play interaction and guidance of the robots. Their study highlights the importance of applying HRI personalization techniques to call the user's attention to scientific subjects. As a result, they found that the robot that performed personalized interactions by calling the visitors by name was better evaluated by the visitors themselves. Also, the robots that carried out a childlike free-play interaction and guided the visitors were the best in attracting attention to scientific explanations.

Research with interactive robotics architectures suggested significant improvement in multimodal interaction, achieved with a simple file management solution (Cortellessa et al., 2018). Multimodal emotional robots are playing an essential role when interacting with children. Results showed that the more human communication resources are demonstrated by the robot, the more the children's confidence in those systems increases (Kessous, Castellano, \& Caridakis, 2010). In educational applications, studies about adaptive robots are recent and the authors report encouraging findings in their usage (Gao, Barendregt, \& Castellano, 2017; Jones \& Castellano, 2018).

However, little is known about how much these works with robots collaborate to place the teacher in a comfortable and active role in planning the activity. This is pointed as an issue to be enhanced in the area (Johal et al., 2018).

\section{Pedagogical Model}

The pedagogical model is based on constructivism, as the educational robotics in general (Kafai et al., 2017). The tutor (in this case the NAO robot, but can be any social robot) plays the main role in the interaction and measures by questions how much the student is rightly constructing its knowledge. The questions can consider objects to be handled by the robot and stories of daily problems to be addressed, characterizing the constructivism.

The ideal scenario is to use this system as practical exercise fixation after the regular classes about the topics' concepts. Each meeting between robot and student is called a session. During a session, the robot presents a concept to the student and evaluate if he/she has understood this explanation by asking questions. For that, every topic that aims to be addressed needs to be registered in the system. The topics have concepts - which is the topic explanation - and as many questions regarding this concept as the designer want to be approached. It is mandatory to divide the questions into five levels of difficulty and at least one question per level in order to guarantee content adaptation. Each session follows the same scheme divided in three phases: Welcome Dialogue, Content Approaching and Closure Dialogue. 
In the first meeting, the Welcome Dialogue phase will recognize the student's face and insert it into the users database. If the student is already registered, all his/her information is recovered to be used in the following conversation. Content adaptation is mapped in the Content Approaching phase. The topics' concept is discussed followed by a random number of questions defined by the designer. The questions are chosen in the difficulty level set by the adaptive function in an instant $t$ of the interaction. The instant $t$ is considered the time to realize a task and it may vary from one task to another. Finally, in the Closure Dialogue, the robot makes a content summary to the student about what was approached in the session. As a feedback, some student's skills, such as average time to respond and correct answers rate, are reported and discussed by the robot. Additionally, some tips about how to improve or keep these skills for the next sessions are presented.

The computational mapping of this methodology is achieved by coding, which is a very unclear process for those who do not have programming knowledge. A graphical interface is presented in the next sections aiming to bring regular teachers closer to robotic solutions for education. The challenge in modeling the contents by following the presented guidelines is a secondary contribution of this research, since it also stimulates the teachers' creativity, pedagogical skills and motivation for new technologies to enclose their methodologies.

\section{Graphical User Interface}

The proposed GUI was implemented to operate over a cognitive adaptive system. Its code can be accessed in the project GitHub website ${ }^{5}$. The adaptive function's goal is to make the interactions as attractive as possible to the student, based on the indicators read along the session. The designer only needs to set up some variables in the GUI (detailed in Subsection 4.4).

The framework PyQt4 ${ }^{6}$ was used to the GUI's development. It facilitates the integration with the architecture that is also implemented in Python language. The software is preferably configured for 14 inches monitors and runs in the same window all the time. The system functionalities are handled in the bottom section of this window by changing the tabs, as detailed in the next subsections. For detailed technical development of each module, please check (Tozadore et al., 2017).

In Figure 2, the system interface is shown separated in two sections. The section (1) is related to the activity summary - highlighted in green - is fixed and the designer can use and the section (2) - highlighted in blue - to configure the activity by each functionality. The activity summary (1) is composed of the main menu buttons on the left of this figure, whereas the activity properties in the middle and an activity picture on the right.

Designers need to be registered in the system and sign in into the software for issues tracking. All their actions are registered and can be accessed whenever one wants. Along the software usage, all information is stored in files that can be reused in other activities or shared through storage devices and networks.

\footnotetext{
${ }^{5}$ https://github.com/LAR-Educational/Architecture_v2_0/tree/master/Arch_2_1/GUI

${ }^{6}$ https://www.riverbankcomputing.com/software/pyqt/download
} 


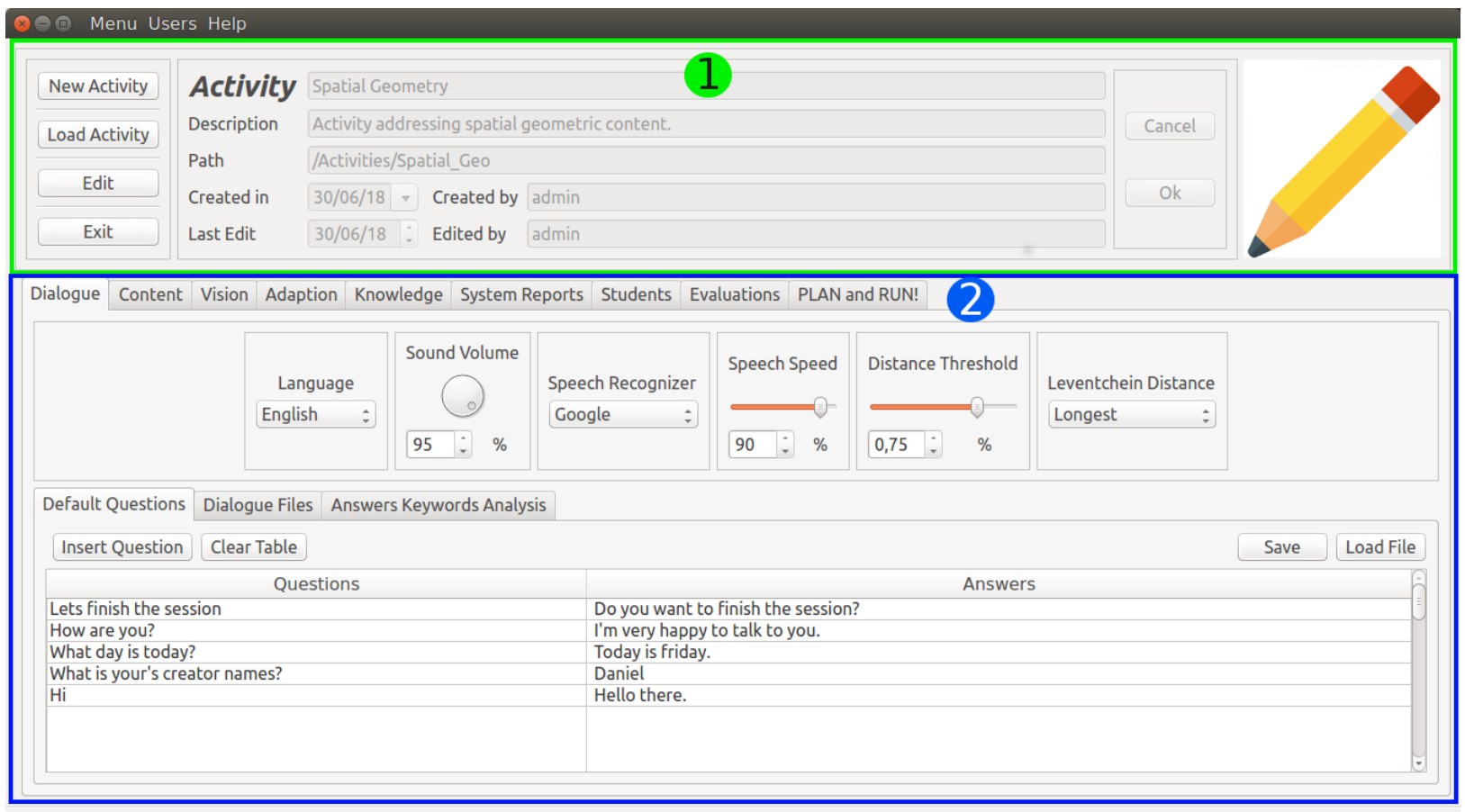

Figure 2: System interface divided in: 1 - the activity summary (fixed); and 2 - the functionalities tab.

The system is divided into the following functionalities, coded in the corresponding tabs.

\subsection{Dialogue}

In the Dialogue tab, it is possible to configure the system variables that will control the verbal interaction. In this project, all dialogues and expected answers must be registered in the system. This is mandatory because the system uses these sentences to compare their distance to the student answers and, based on a threshold set by the designer, judge if the answer is right or wrong. The chosen comparison algorithm is the Levenshtein distance, which is very used in Natural Language Processing techniques and DNA comparison.

In the Dialogue functionality tab (Figure 2) it is possible to set the components: Language (English or Portuguese), Volume (0 to $100 \%$ ), Speech Recognition Method (Google Recognition or NAO's Default), Robot's Speech Speed (0 to $100 \%$ ), Levenshtein Distance Threshold (0 to 1) and Levenshtein Distance Method (Longest or Shortest). The bottom section is a frame with tabs responsible for control the tables: "Default Questions" to register possible questions that can be made to the robot in any part of the interaction; "Conversation Set Up" to write Welcome and GoodBye dialogues; and "Answers Keyword Analysis" to set students vocabulary for affirmation, negation and doubt.

\subsection{Content}

The Content tab allows to create and manage the topics according to the adopted Pedagogical Model (Section 3). The activities can have as many topics as the designer wants and it is also possible to import topics from other activities. The content is defined by topics the designer aims to address during the content approaching phase. As can be seen in Figure 3, topics are easily inserted by clicking on the "New Subject" button. Already registered topics are handled in the corresponding combo box.

Topics' concepts are inserted or displayed in the concept field. The concept is the topic definition and it is exactly what the robot will explain about this topic to the student. Due to resource limitations, the robot exclusively counts on verbal explanation to address the contents. However, future works will include adding a visual display, such as tables or screens, to increase 
the explanation experience. In the bottom of the content tab, the designer registers the questions of every difficulty level. It is mandatory to register one question of each level and desirable to have as many as possible.

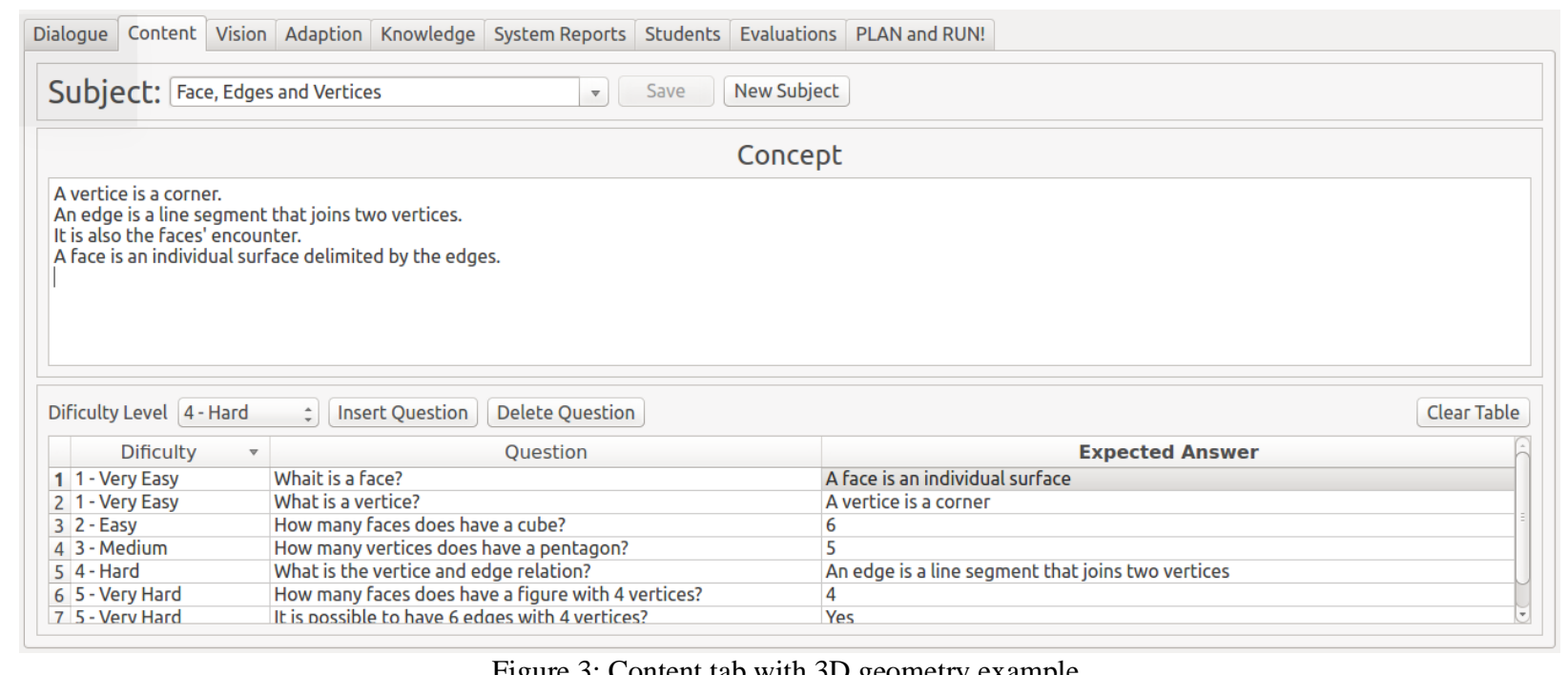

\subsection{Vision}

The Vision module is optional in the activities. It is responsible for recognizing and classifying the objects using Machine Learning methods. The implemented methods are Multilayer Perceptron (MLP) Networks, K-Nearest Neighbor (KNN), Support Vector Machines (SVM) and Convolutional Neural Networks (CNN). Objects database creation or reuse is required for every activity that uses the vision module. Methods train and validate the databases with specific speed and accuracy. For instance, the KNN and SVM have fast training time (time $<10$ seconds) and perform it just before the sessions, whereas the MLP and CNN require more training time (time $>5$ hours). More details about advantages and setbacks in using these methods can be found in (Tozadore \& Romero, 2017). The vision tab has a section to manage the database, a section to manage the classification methods (middle) and a section for samples visualization (right), as can be seen in Figure 4.

\subsection{Adaption}

The Adaptive module aims to change the robot's behavior according to the observed student's indicators, expressed by body language and verbal answers. These indicators were divided into three main groups regarding the measures of Attention, Communication and Learning. 


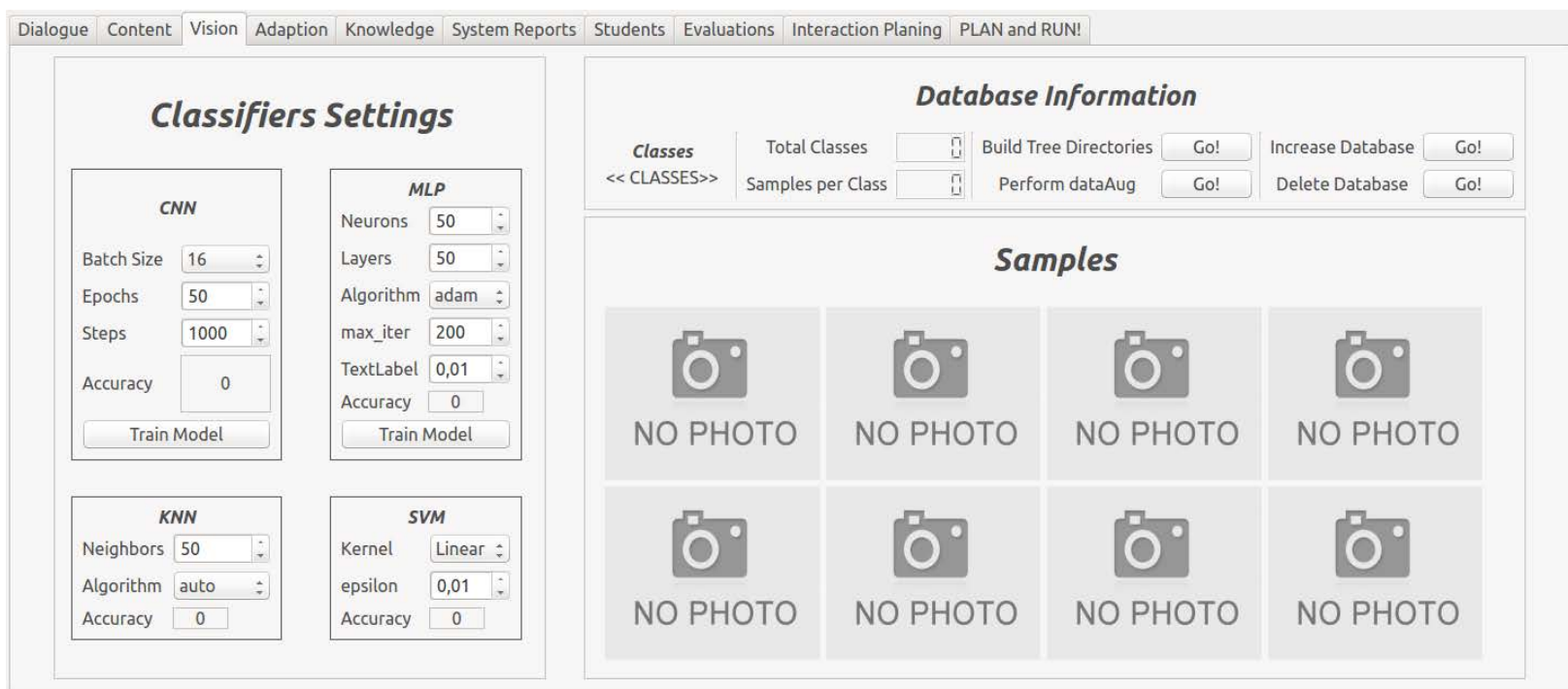

Figure 4: Vision tab view. The available methods and their parameter in the left section and the database information in the right section.

They are: Face gaze for the Attention; users' Emotions for Communication; and Right/Wrong answer and Time to answer the proposed exercises for the Learning group. The average of the objective measures of each group result in a final major value of the class, named as $\alpha$ to Attention, $\beta$ to Communication and $\gamma$ to Learning.

Table 1: Caption table 1.

\begin{tabular}{|c|c|c|}
\hline Attention $(\alpha)$ & Communication $(\boldsymbol{\beta})$ & Learning $(\gamma)$ \\
\hline Face gaze & Number of words & Right/wrong answer \\
\hline & Emotions & Time to answer \\
\hline
\end{tabular}

Each major value is calculated by their respective measures' tolerance normalization between 0 and 1 . The vectors correspond to the activation value for each weight in the $F_{a d p}$ calculation in Equation (1). The maximum limit for the major values is 1 and when applied to the $F_{a d p}$ equation, they will fully activate their corresponding weight from the class. 0 means that there was no detection of this class activity in the instant $t$. Thus, this value corresponding weight will not contribute to the $F_{a d p}$ in the instant $t+1$. In other words, the classes major values $\alpha, \beta$ and $\gamma$ mean how much their respective class is being critical (from 0 for none to 1 for maximum) in the specific instant $t$, whereas the weights potentialize how much their respective class is contributing to the $F_{a d p}$ calculation and their values are the same all along the session. It is worth to notice that the sum of the weights should not be greater than one in order to fit the interval from 0 to 1 for the adaptive function $F_{a d p}$.

The adapted robot's behavior, denoted as $\Psi$, is an iterative function calculated by its last value added by the function $F_{a d p}$, as shown in Equation (1):

$$
F_{\text {adp }}(t)=w_{\alpha} * \alpha(t)+w_{\beta} * \beta(t)+w_{\gamma} * \gamma(t)
$$

where $\boldsymbol{t}$ is the instant in which the robot is approaching one question of a topic

The $F_{a d p}$ is a function to adapt the resulting robot's behavior $\Psi$, trying to optimize the interaction engagement and learning rate. The parameters $\alpha, \beta$ and $\gamma$ are the group activation function outputs and the $w_{\alpha}, w_{\beta}$ and $w_{\gamma}$ are the corresponding weights, set by the designer before the beginning of the session.

In Equation (2), the calculation of the robot's behavior state at the instant $\boldsymbol{t}$ is presented. The $\Psi(0)=3$ state guarantees the system starts in neutral behavior. 


$$
\Psi(t)=\left\{\begin{array}{ll}
3, & \text { if } t=0 \\
\Psi(t-1)+1, & \text { if } F_{\text {Adp }}(t) \geq 0,66 \\
\Psi(t-1)+0, & \text { if } 0,66<F_{\text {Adp }}(t)<0,33 \\
\Psi(t-1)-1, & \text { if } F_{\text {Adp }}(t) \leq 0,33
\end{array} \quad, t \in \mathbb{N}\right.
$$

Regarding the adaptive tab in the GUI, the values $\alpha, \beta$ and $\gamma$ are set in the corresponding section as can be seen in Figure 5.

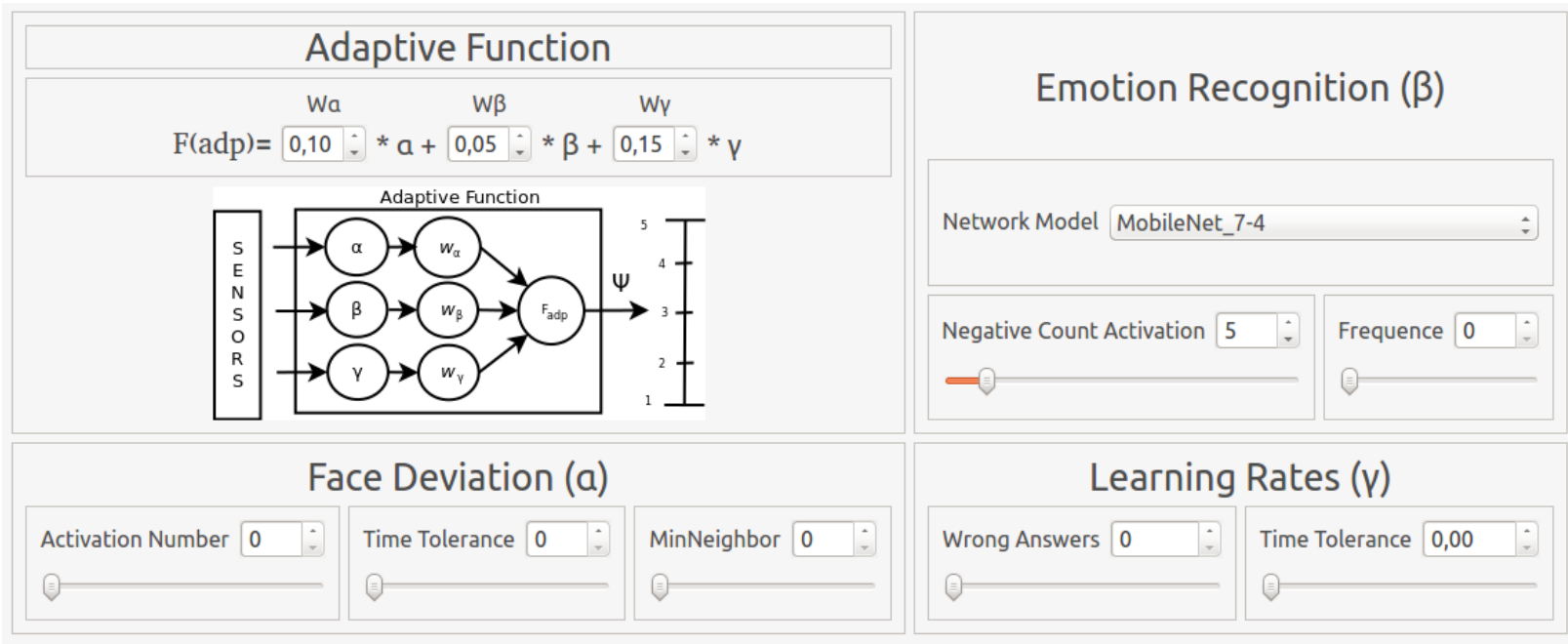

Figure 5: Adaptive tab view.

The face gaze is handled by a Haar Cascade algorithm in which the "MinNeighbor" and the "Time Tolerance" are considered to count a deviation (Viola \& Jones, 2001). The "Activation Number" is the sum of the deviation counter in the end of instant $t$ and is the variable that will be normalized to activate the weight $w_{\alpha}$. The communication vector is the sum of the emotions recognized and number of words spoken by the student. The average of both measures is normalized and used to activate the weight $w_{\beta}$. Six different models of Convolutional Neural Networks were compared to classify the emotions, as it can be found in (Tozadore, Ranieri, Nardari, Guizillini, \& Romero, 2018). "Counter Threshold" is the number of negative emotions detection to maximum activation. The number of words is counted by the Dialogue module. Leaning vector measures are provided by the Dialogue module. Its activation value is also the average of the "Wrong Answers" and "Time to Response" thresholds, which will influence the weight $w_{\beta}$.

\subsection{Student Database}

Students database stores personal information, such as, first name, surname, birthday date, school year, and 8 personal preferences as sport, dance, team, music, toy, hobby, game and food. The system searches in the Knowledge database about the choices of the students and uses them in talks as it is necessary, aiming to simulate long-term relations. These talks are performed according to the interaction flow (programmed in Section 4.8) or triggered by the adaptive module if it detects signs of students' low engagement. This information is collected by the robot in its first meeting with the student in short dialogues. Insertions, modifications or deletions can be made in the User tab, as shown in Figure 6. 


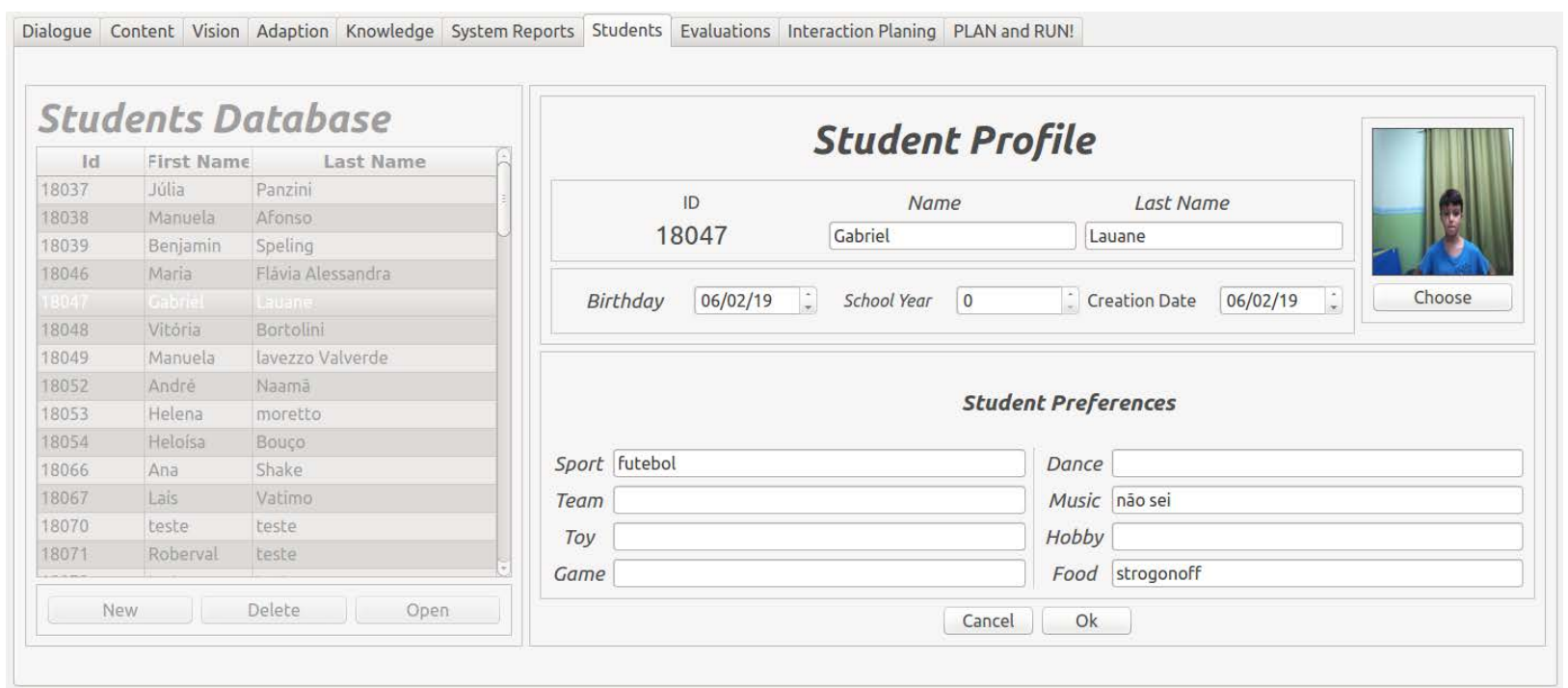

Figure 6: Students window. On the left side of the screen is the students database and in the right section the selected student's information is shown.

\subsection{Knowledge}

Similarly, in the knowledge database of the system all the nouns definitions are recorded through manual insertion or through automatic searched by the system on the internet. It is possible to insert content into the system through the Knowledge tab (Figure 7) or the system can search by them during the interaction. In case of new entries, the system searches in the Wikipedia website ${ }^{7}$ through a python API. A small language processing technique is employed to extract the noun abstract. The personal database stores information created about the robot "personal life" is also stored, such as how old he/she is, how many brothers and sisters he/she has, what is his/her name and so on. Previous studies showed significantly higher rapport building when humanizing the robot (Pinto, Tozadore, \& Romero, 2015).

\subsection{Evaluation}

Once the sessions are individual, it can be searched by the student that performed the activity in the evaluation database and this activity evaluation summary will be shown in the Evaluation Tab (Figure 8). The evaluation overview shows the Student Name, Execution Date, Supervisor (the designer that performed this activity), the time it was executed and a student picture at the section top. It also shows information that are relevant to the session, for instance, the total number of content questions asked by the robot, maximum number of attempts per question, student correctness rate and observations added by the supervisor after the sessions ended. In the right section, a multi-tab frame allows to navigate through the Topics Validation, a Timeline Session Evolution, the adaptive system metrics auto analysis and some pictures taken by the robot during the session.

\footnotetext{
${ }^{7}$ www.wikipedia.com
} 


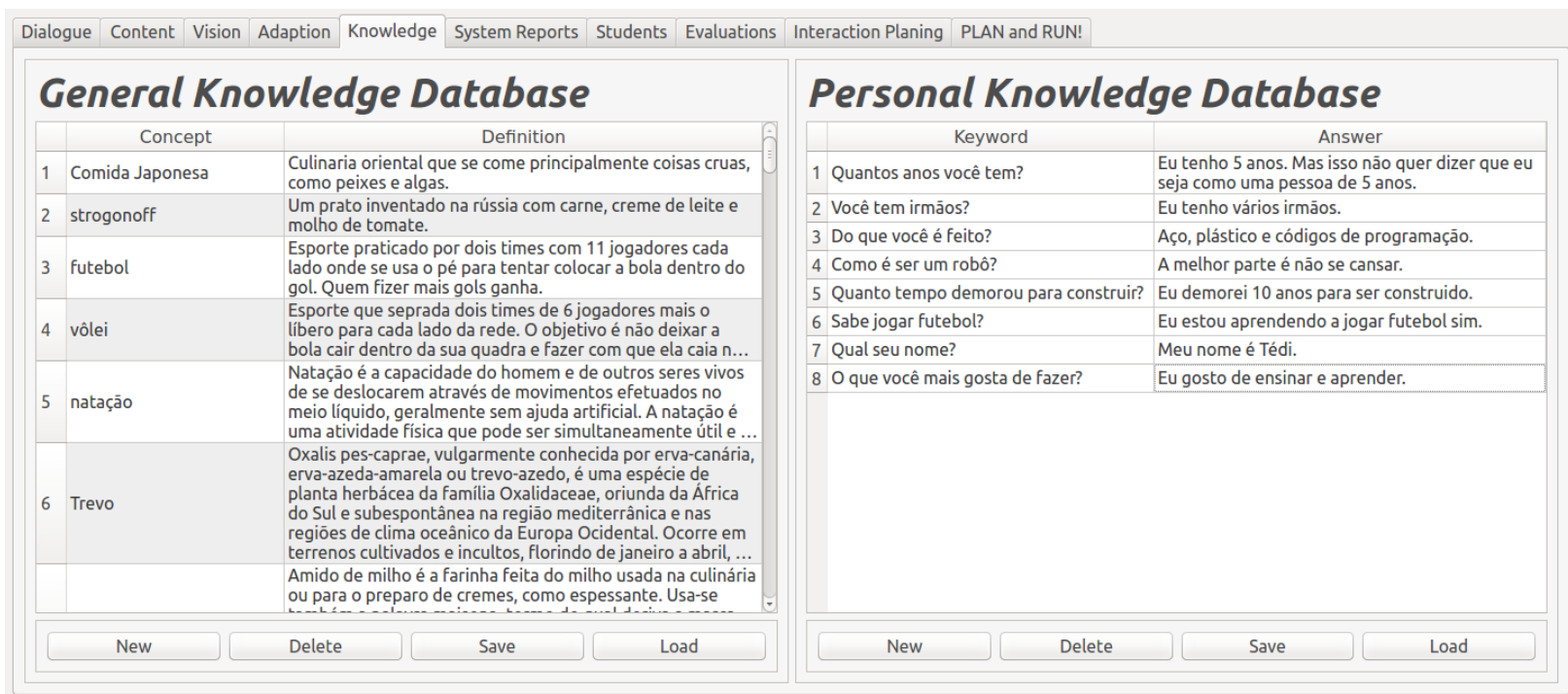

Figure 7: Knowledge database management. The left section is the general knowledge entries and in the right section the robot's "personal information".

The Topics Validation tab recovers all the content information exchanged between robot and the student along the corresponding session for validation. The supervisor needs to manually observe and validate if the system automatically classified the student answers right or wrong. Afterwards the questions validation, the system can generate an evaluation regarding its accuracy and the student performance.

This information about system and student performance in the interaction is shown as charts in the Information tab. It is considered one of the advantages of this system, because it is a helpful type of diagnostic about the student difficulty, in which the teachers can visualize the critical learning areas through charts and prepare the next activities focused in last sessions point of weakness.

\subsection{Interaction Planning}

The Interaction Planning tab is responsible for programming the interaction flow. It means the sequence of commands that will be executed by the robot during the session. There are three types of actions that the robot can perform, as shown in the left boxes of Figure 9: Content approach, Personalized talks and Extra actions.

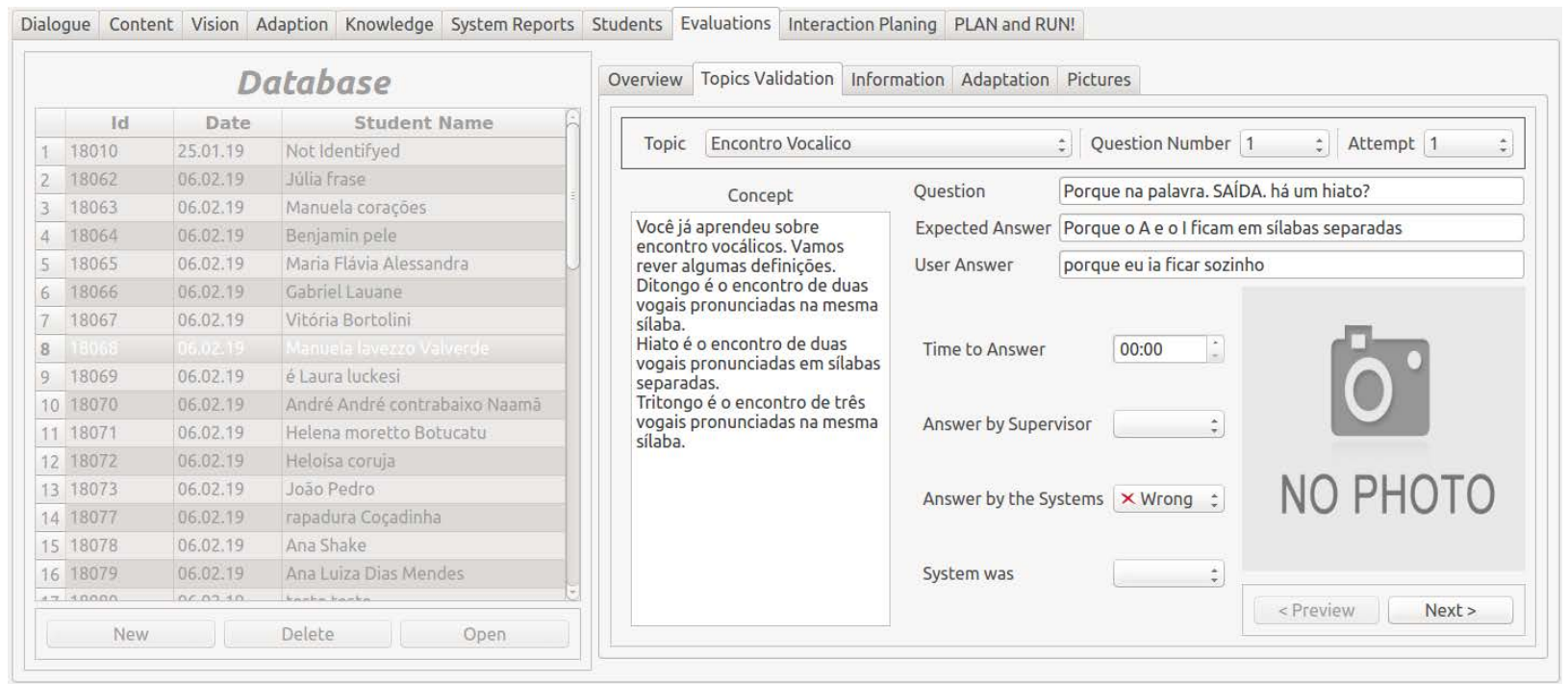

Figure 8: Evaluation tab. The left section is the evaluations database and the right section shows the selected evaluation's information. 


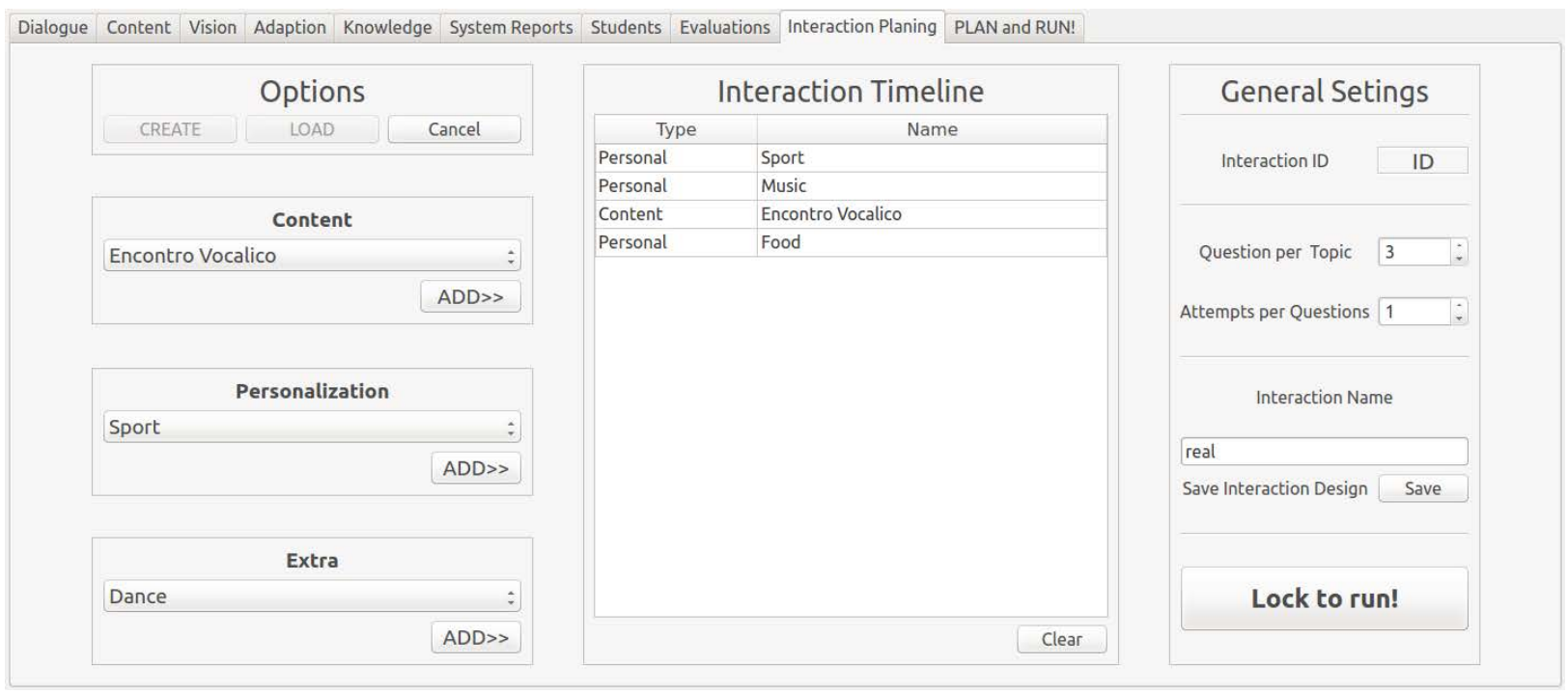

Figure 9: Interaction tab. The sequence of interaction steps is chosen by the designer.

In the Content approach actions, the robot will ask about the number of questions as set in the number of questions of the selected content. For each question, the student will have the maximum number of attempts set in the attempts number. All available contents to be approached are registered in the system through the content tab.

The Personalized talks are made of a small dialogue, in which the robot will ask the student what is his/her preference about the selected theme. The system searches for the student's answer in the local knowledge database and talks about it, if found. Otherwise, the system searches in a Wikipedia page, as explained in Section 4.6. If this conversation has already happened before, the system recovers the student's preferences and takes the same action as if he/she had answered that preference. These preferences can be manually set for each user, as shown in Figure 6.

The Extra actions include Dances, Games or any other performances that are programmed in the robot behavior set. The actions are added to the timeline table, in the center of the screen, and the session will flow in this exact order.

\subsection{Run}

After setting the described configurations, the Plan and Run tab (Figure 10) provides the session high-level scheme to the designer. In the left panels, the robot IP address and the robot communication port are required to initiate the session. It is possible to observe in execution time some system variables that change along the session, such as the adaptive function parameters, the robot behavior $\Psi$, previous values of robot behavior, user emotion read by the system, among others.

After these settings, the "Start" button is enabled. By starting the session, some variables that change over time are tracked in the bottom left section of the window in corresponding frames. The middle section of the screen displays the robot's camera image. Three fields of information tracking are placed below the robot view. They are the expected and understood answers field, that displays the corresponding information, and the user manual answer, in which is possible to insert the answers through the keyboard, if this option is enabled in the dialogue tab.

In the right panels, it is possible to force the system flow, jumping through the questions and topics - which is used is extreme cases - and take pictures and save videos from the robot's camera. In the bottom of these sections is the field corresponding to the robot's talks, that shows what the robot is speaking. 


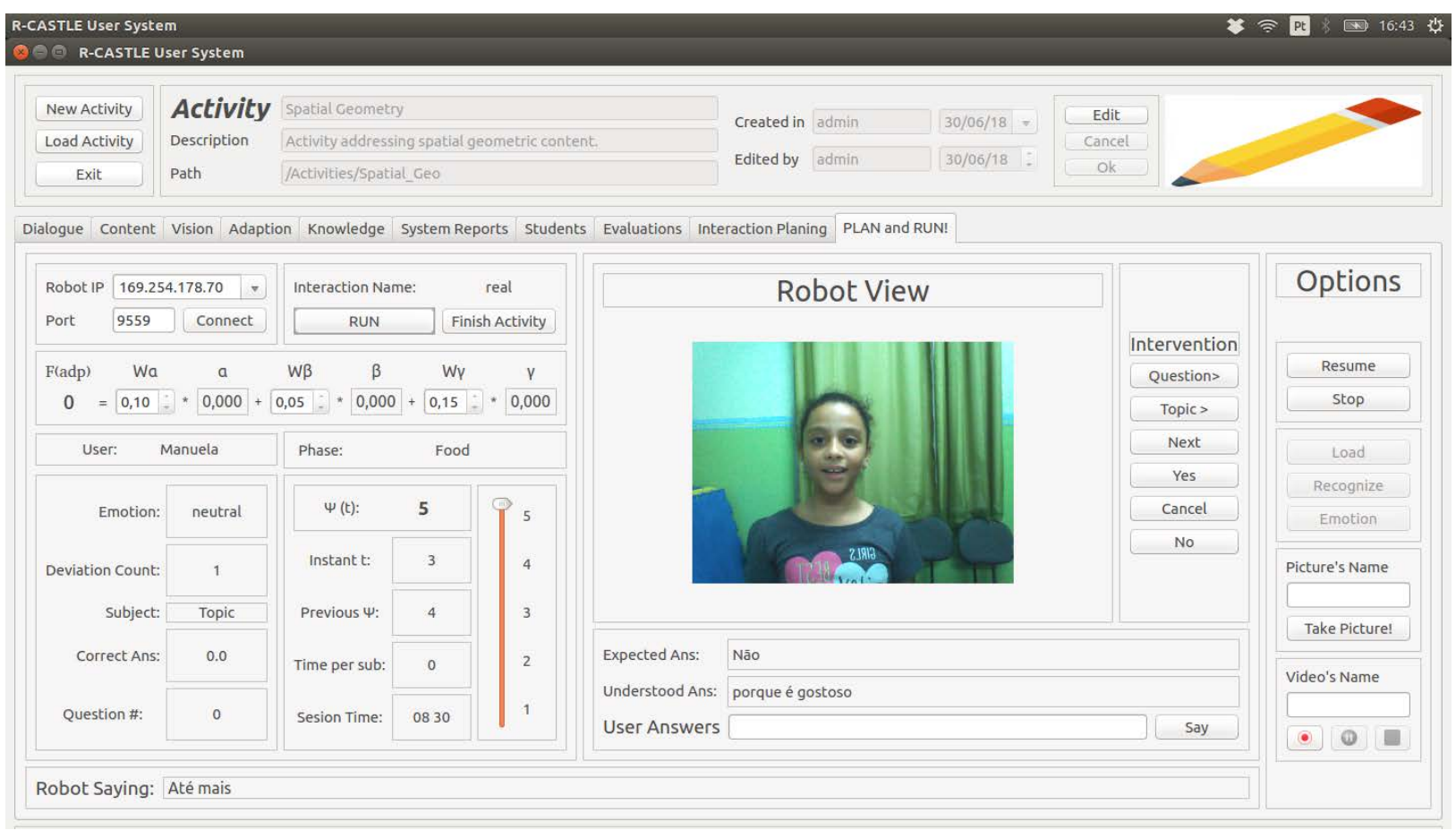

Figure 10: Plan and Run tab: The monitored variables in the left, the robot view in the middle and the extra actions panel in the right.

\section{Teachers' perception}

In order to analyze the teachers' first perception about the system's potential, 14 teachers from the elementary school "Oca dos Curumins", located in São Carlos city, participated in a 50 minutes demonstration about the GUI. The presentation approached the GUI components that are more relevant to the teacher's usage. As said previously, in general, it is expected that the teachers are more interested in some functionalities of the system such as the Content programming, the Student profiles and the Evaluations performed, than other GUI functionalities that may contain information more relevant to the developers, such as the vision, adaptation and system's log. During the presentation, explicitly mentioning the GUI advantages or disadvantages was avoided, to avoid inducing the participants (the teachers) to a bias in their perception about the whole system. In other words, only functionalities of the system and their use were explained, letting the perception of how the system can be useful to their own judgment. The teachers that wanted to interact with the interface could do so at the end of the explanation.

The aim was to evaluate the system strength of generalization, by giving an overall explanation, supporting the principle that the methodology can cover the areas of knowledge of the teachers. Finally, 9 out 14 of those teachers ( 2 males and 7 females) aged in average 43.2 (SD 12.7) years old, answered a 5-Likert Scale questionnaire about the GUI. The Likert Scale is very common in research about marketing that aim to know how much the customers would enjoy some products' characteristics. The answer's possibilities were a scale from 1 to 5 , where 1 means Absolutely Nothing and 5 means For Sure for every item. Thus, 3 is the neutral score. The following questions were asked:

1. How much are you familiarized with technology?

2. How much do you think this application can support you in your class activities?

3. How much do you think it will be easy to create activities in this tool?

4. How much do you think this application has powerful to become a regular tool in teaching?

5. How much do you think this methodology is efficient for content approaching? 
6. How much time do you think it is necessary to get used with this GUI?

7. How much do you think this methodology can enhance the students' learning?

8. How much do you think that technological methodologies are more efficient compared to the traditional ones?

The average score for each item can be seen in Figure 11. Beyond the 8 questions above, 2 open questions were optional. The points raised in these questions were computed and their number (n) of occurrence is shown below:

A. What are the system advantages from your point of view?

Answers: Motivation ( $n=5)$, Adaptation $(n=3)$, Technological Interaction $(n=3)$, Teacher Support ( $\mathrm{n}=2)$, Data Handling $(\mathrm{n}=1)$.

B. What are the system disadvantages from your point of view?

Answers: Number of Variables ( $n=5)$, Robot Cost $(n=2)$, Teachers' Adaptation $(n=1)$.

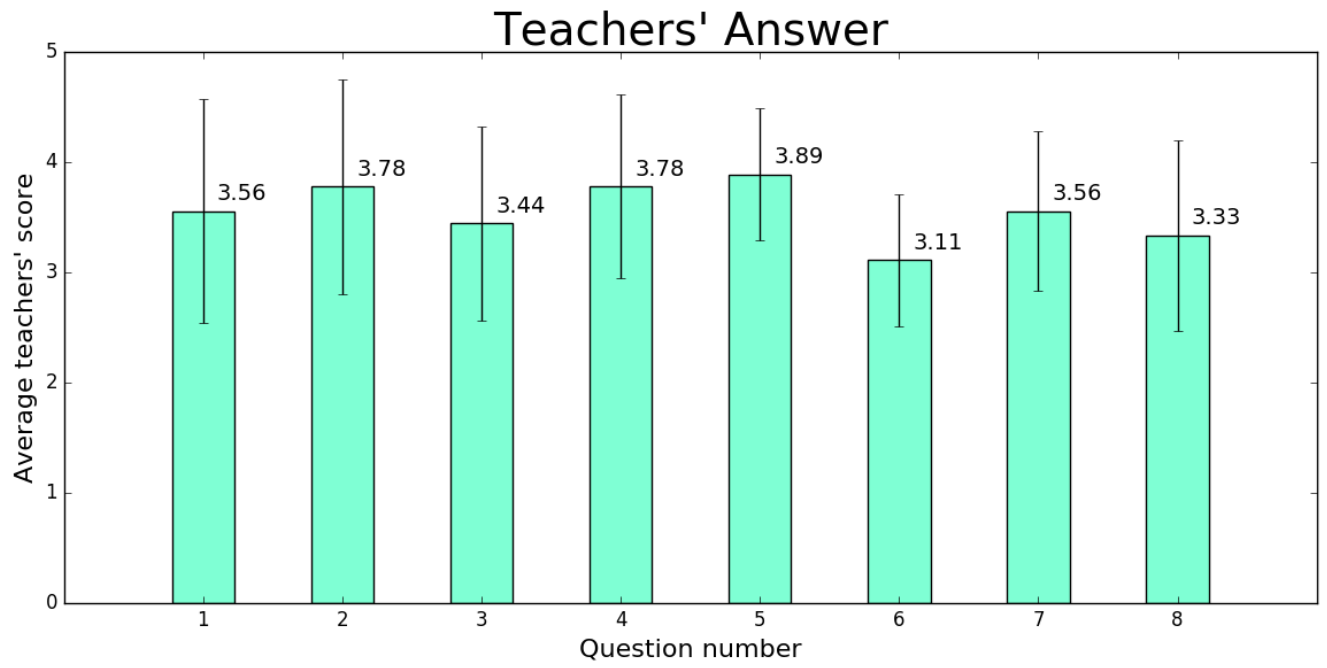

Figure 11: Teachers’ score average for Likert Items.

By analyzing the results, a curious fact noted was that they scored the facility in programming of the exercises in the GUI (Item 3) lower than they claimed they are familiarized with technology (Item 1). However, they show they understood the GUI's potential in supporting their classes (Item 2) with one of the highest averages of 3.78. The same score as they rated the system potential in becoming a regular tool in teaching (Item 4).

The highest score was achieved by the item that asked about the methodology efficiency in content approaching (Item 5). One fact that may explain this point is the similarity of the system pedagogical model with the regular methodology the school have. Thus, to adapt their regular methodology to the system would not be a difficulty for them.

They rated the Item 6, regarding the time to get used with the system, with a score of 3.11, which is very close to the neutral score. It suggests that they would take the same time to get used with this system as they would have to get used with another educational methodology.

The most surprising score was at Question 8, about this methodology efficiency compared to regular methods. It was slightly above the neutral score (3.33). Assuming the difficulty in changing consolidate methods, this score may raise a point in the sense of how teachers are 
noticing the necessity in starting to migrate from non-technological methods to innovating solutions.

Regarding the open questions, the most cited advantage of the system was the motivation that the robots provide, which is not novelty as shown by several researches in the area (Leite et al., 2013). The system's behavior adaptation was also noted as a strong point by some teachers, and only one participant claimed the facility in data handling as a notable advantage. Many of the participants pointed the amounts of variables that the system deals with as a potential problem to their usage. They said the adaptation system and its weight management is something they would take more time to understand. The robot cost was also cited as a disadvantage, because the robot used in the demonstration was the NAO robot, acquired from a FAPESP project by the host University. Nonetheless, the system can run along any interactive device that has cameras and microphones.

In summary, all the evaluated items had a score above the neutral score (3), but few of them got close to have an average in 4 . This fact leads to believe that the system's potential was not fully perceived by all the participants that answered the questionnaire. Potentially, because they need more time for getting familiarity with the GUI and to know all the system's practices. However, the system advantages planned by the researchers were cited at least by one of the professors that answered the questionnaire. According to the teachers' statements themselves, perhaps more familiarization with the interface by using and testing it may result in perceptions more accurate about how this system can actually support their classes.

\section{Conclusion}

This paper showed the proposed interface for educational content management, providing autonomously robot behavior and questions difficulty adaptation. The system tries to simulate rapport building by keeping a database of students' preferences about daily subjects to interact with them along the session. Through face recognition, the system can support long-term interactions, retrieving the interactions optimization parameters for each user and approaching the programmed contents according to these variables. Following the adopted methodology, it is possible to set up the robotic system to interact in almost every area of elementary school subject. The proposed GUI is an alternative to work around the problem of lack of familiarization with social robots by non-programmers. After a short presentation of the interface to a group of regular teachers from elementary school, it was possible to identify their feedback as positive in the application of the proposed system as a pedagogical tool. All teachers of this study perceived some potential in using the presented system that can be enhanced by the time of usage and familiarization with the system variables. Future work includes performing several activities with the robot and a group of teachers and students to investigate the impact of the proposed system in their performance along their regular academic year.

\section{Acknowledgements}

This study was financed by the Coordenação de Aperfeiçoamento de Pessoal de Nível Superior Brasil (CAPES) - Finance Code 001, the Fundação de Amparo à Pesquisa do Estado de São Paulo (FAPESP) and the Conselho Nacional de Desenvolvimento Científico e Tecnológico - Brasil (CNPq). 


\section{References}

Belpaeme, T., Kennedy, J., Ramachandran, A., Scassellati, B., \& Tanaka, F. (2018a). Social robots for education: A review. Science Robotics, 3(21), DOI:10.1126/scirobotics.aat5954 [GS Search]

Belpaeme, T., Vogt, P., Van den Berghe, R., Bergmann, K., Göksun, T., De Haas, M. \& Papadopoulos, F. (2018b). Guidelines for designing social robots as second language tutors. International Journal of Social Robotics, 10(3), 325-341. DOI: 10.1007/s12369-018-0467-6 [GS Search]

Benitti, F. B. V. (2012). Exploring the educational potential of robotics in schools: A systematic review. Computers \& Education, 58(3), 978-988. DOI: 10.1016/j.compedu.2011.10.006 [GS Search]

Clabaugh, C., Tsiakas, K., \& Mataric, M. (2017). Predicting preschool mathematics performance of children with a socially assistive robot tutor. In Proceedings of the Synergies between Learning and Interaction Workshop@ IROS, Vancouver, BC, Canada (pp. 24-28). [GS Search]

Cortellessa, G., Fracasso, F., Sorrentino, A., Orlandini, A., Bernardi, G., Coraci, L. \& Cesta, A. (2018). ROBIN, a telepresence robot to support older users monitoring and social inclusion: development and evaluation. Telemedicine and e-Health, 24(2), 145-154. DOI: 10.1089/tmj.2016.0258 [GS Search]

Gao, A. Y., Barendregt, W., \& Castellano, G. (2017). Personalised human-robot co-adaptation in instructional settings using reinforcement learning. In IVA Workshop on Persuasive Embodied Agents for Behavior Change: PEACH 2017, August 27, Stockholm, Sweden [GS Search]

Goodrich, M. A., \& Schultz, A. C. (2008). Human-robot interaction: a survey. Foundations and Trends in Human-Computer Interaction, 1(3), 203-275. DOI: 10.1561/1100000005 [GS Search]

Johal, W., Castellano, G., Tanaka, F., \& Okita, S. (2018). Robots for learning. International Journal of Social Robotics, 293-294. DOI: 10.1007/s12369-018-0481-8 [GS Search]

Jones, A., \& Castellano, G. (2018). Adaptive robotic tutors that support self-regulated learning: A longer-term investigation with primary school children. International Journal of Social Robotics, 10(3), 357-370. DOI: 10.1007/s12369-017-0458-z [GS Search]

Kafai, Y., Sawyer, C. I. R., Papert, S., Harel, S. C. I. I., Papert, S., \& Duval, E. (2017). Technology and theories of learning. Technology Enhanced Learning: Research Themes, 17(1), 169. [GS Search]

Kessous, L., Castellano, G., \& Caridakis, G. (2010). Multimodal emotion recognition in speechbased interaction using facial expression, body gesture and acoustic analysis. Journal on Multimodal User Interfaces, 3(1-2), 33-48. DOI: 10.1007/s12193-009-0025-5 [GS Search]

Leite, I., Martinho, C., \& Paiva, A. (2013). Social robots for long-term interaction: a survey. International Journal of Social Robotics, 5(2), 291-308. DOI 10.1007/s12369-013-0178-y [GS Search]

Lucas, G. M., Boberg, J., Traum, D., Artstein, R., Gratch, J., Gainer, A. \& Nakano, M. (2018, February). Getting to know each other: The role of social dialogue in recovery from errors in social robots. In Proceedings of the 2018 ACM/IEEE International Conference on HumanRobot Interaction (pp. 344-351). ACM. DOI: 10.1145/3171221.3171258 [GS Search] 
Mubin, O., Stevens, C. J., Shahid, S., Al Mahmud, A., \& Dong, J. J. (2013). A review of the applicability of robots in education. Journal of Technology in Education and Learning, 1(209-0015), 13. [GS Search]

Murray, T. (1999). Authoring intelligent tutoring systems: An analysis of the state of the art. International Journal of Artificial Intelligence in Education (IJAIED), 10, 98-129. [GS Search]

Pinto, A. H., Tozadore, D. C., \& Romero, R. A. (2015, October). A question game for children aiming the geometrical figures learning by using a humanoid robot. In 2015 12th Latin American Robotics Symposium and 2015 3rd Brazilian Symposium on Robotics (LARS-SBR) (pp. 228-233). IEEE. DOI: 10.1109/LARS-SBR.2015.62 [GS Search]

Platz, M., Krieger, M., Niehaus, E., \& Winter, K. (2018). Suggestion of an E-proof Environment in Mathematics Education. In Classroom Assessment in Mathematics (pp. 107-120). Springer, Cham. DOI: 10.1007/978-3-319-73748-5_8 [GS Search]

Rivas, D., Alvarez, M., Velasco, P., Mamarandi, J., Carrillo-Medina, J. L., Bautista, V. \& Huerta, M. (2015, February). BRACON: Control system for a robotic arm with 6 degrees of freedom for education systems. In 2015 6th International Conference on Automation, Robotics and Applications (ICARA) (pp. 358-363). IEEE. DOI: 10.1109/ICARA.2015.7081174 [GS Search]

Shiomi, M., Kanda, T., Ishiguro, H., \& Hagita, N. (2006, March). Interactive humanoid robots for a science museum. In Proceedings of the 1st ACM SIGCHI/SIGART conference on Humanrobot interaction (pp. 305-312). ACM. DOI: 10.1145/1121241.1121293 [GS Search]

Spaulding, S., Chen, H., Ali, S., Kulinski, M., \& Breazeal, C. (2018, July). A Social Robot System for Modeling Children's Word Pronunciation: Socially Interactive Agents Track. In Proceedings of the 17th International Conference on Autonomous Agents and MultiAgent Systems (pp. 1658-1666). International Foundation for Autonomous Agents and Multiagent Systems. [GS Search]

Tozadore, D., Ranieri, C., Nardari, G., Guizilini, V., \& Romero, R. (2018, October). Effects of Emotion Grouping for Recognition in Human-Robot Interactions. In 2018 7th Brazilian Conference on Intelligent Systems (BRACIS) (pp. 438-443). IEEE. DOI: 10.1109/BRACIS.2018.00082 [GS Search]

Tozadore, D. C., Valentini, J. P. H., de Souza Rodrigues, V. H., Vendrameto, F. M. L., Zavarizz, R. G., \& Romero, R. A. F. (2018, November). Towards Adaptation and Personalization in Task Based on Human-Robot Interaction. In 2018 Latin American Robotic Symposium, 2018 Brazilian Symposium on Robotics (SBR) and 2018 Workshop on Robotics in Education (WRE) (pp. 383-389). IEEE. DOI: 10.1109/LARS/SBR/WRE.2018.00075 [GS Search]

Viola, P., \& Jones, M. (2001). Rapid object detection using a boosted cascade of simple features. CVPR (1), 1, 511-518. [GS Search] 